\title{
KONZEPTUELLE METAPHER AM BEISPIEL DER DEUTSCHEN BÖRSENBERICHTE IM RAHMEN DES FACHSPRACHENUNTERRICHTS DER LODZER GERMANISTIK
}

\section{CONCEPTUAL METAPHER IN THE FRAMEWORK OF THE SPECIALIZED LANGUAGE LESSONS AT GERMAN STUDIES IN LODZ ON THE EXAMPLE OF THE GERMAN STOCK MARKET REPORTS}

Mit der Entwicklung der Korpuslinguistik wird der Ruf nach einer auf natürlichen Daten basierenden Analyse von Metaphern in den letzten Jahren immer ausdrucksvoller. Diesem Trend folgend, geht der vorliegende Text, basierend auf der kognitiven Metapherntheorie, anhand der Korpora auf der Grundlage einiger deutschen Online-Börsenzeitungen, der Frage nach, wie das Konzept des Börsenparketts durch Metaphern im deutschen wirtschaftlichen Diskurs strukturiert und den Germanistikstudierenden auf zugängliche Weise darzustellen ist. Von den Suchergebnissen wurden die meist auftretenden Metaphern analysiert. Dann wurden diese metaphorischen Ausdrücke nach den Ursprungsbereichen sortiert. Das Untersuchungsergebnis zeigt: die Konzeptualisierungen von den Börsenprozessen sind dem Laien nicht fremd. Sie beruhen auf Grundkenntnissen zur Welt und gemeinsamen Erfahrungen der Menschen, und sind zu einem festen Bestandteil des menschlichen Konzeptsystems geworden.

Schlüsselwörter: konzeptuelle Metapher; fachsprachliche Metapher; Börsenberichterstattung; Börsensprache; Konzeptualisierung

With the development of corpus linguistics, the need for natural metaphor analysis based on economic data has become more pronounced in recent years. In line with this trend the following text introduces the concept of a cognitive metaphor. It is

\footnotetext{
* Dr., Łódź, Universität Łódź, Polen.
} 
based on examples from a number of German online stock newspapers, and addresses the issue of transparency of stock exchange markets in German economic discourse. The text shows how to easily transfer this knowledge to students of German studies. The most common metaphors were approximated on the basis of conducted research. Then, they were grouped in terms of semantics. The results of the study show that the conceptualization of stock market processes is not familiar to a non-expert. They are connected with common knowledge of the world and shared human experience as well as have become an integral part of the human conceptual system.

Keywords: conceptual metaphor; metaphor in technical language; Stock market reporting; Exchange language; conceptualization; terminology; specialized languages

\section{Einleitung}

Universitäten haben längst ihren hermetischen Charakter verloren, der auf ihrer elitären Funktion beruhte. Die heutigen Universitäten sind oft Zentren der sozialen Entwicklung, Auslöser sozialer, wirtschaftlicher und politischer Veränderungen. Die moderne Universität erhält neben der didaktischen und Forschungsfunktion, die in der Literatur als erste und zweite Mission bezeichnet werden, eine weitere wichtige Funktion im Hinblick auf das Wohlbefinden der Gesellschaft, die Beziehungen zum sozioökonomischen Umfeld aufbaut und entwickelt. Diese Aufgabe wird als dritte Mission der Universität definiert. Die Maßnahmen umfassen die Entwicklung des Angebots der Universität im Bereich der Umsetzung der dritten Mission, beispielsweise durch Programme, die in Zusammenarbeit mit Unternehmen durchgeführt werden und zur Entwicklung von Schlüsselkompetenzen beitragen, die den Bedürfnissen des Arbeitsmarktes, der Wirtschaft und der Gesellschaft entsprechen. Die dritte Mission beruht aber auch darauf, die Studierenden für den Berufsleben vorzubereiten, damit ihre Chancen auf dem Arbeitsmarkt mit dem Hochschulabschluss besser sind. Dementsprechend wird auch das Programm der Lodzer Germanistik auf diese Art und Weise bearbeitet, damit die Absolventen nicht nur die deutsche Allgemeinsprache beherrschen und über das Wissen über die deutschsprachige Literatur, deutsche Kultur oder sprachwissenschaftliche Grundlage verfügen, sondern auch die Grundlagen des Fachwissens und der Fachsprachen beherrschen, was den Einstieg in den Arbeitsmarkt bildet?

Wirtschaftliche Sachverhalte sind ein wichtiger Teil unserer alltäglichen Kommunikation, vor allem in Verbindung mit der Wirtschaft. Das Verständnis dieser Fachsprache und des Fachwissens in diesem Bereich ist daher nicht nur Fachleuten sondern auch Laien wichtig, um im Alltag problemlos existieren zu können (vgl. Hundt 1995, 5). Es entstehen immer mehr sowohl deutsch- als auch polnischspra- 
chige wissenschaftliche Arbeiten im Bereich der wirtschaftsbezogenen Fachsprachenforschung, was noch in 90er Jahren des 20 Jahrhunderts nicht so deutlich betont worden ist. Auf dieses Phänomen wies u. a. Bolten (1991, 71f.) hin:

Obwohl die Fachsprachenforschung in den vergangenen Jahren insgesamt erhebliche Fortschritte verzeichnen konnte, ist die Erforschung der Fach- und Berufssprache Wirtschaftsdeutsch eher stiefmütterlich behandelt worden. Aktuelle Monographien und Handbücher zur Fachsprachenforschung berücksichtigen den Bereich Wirtschaftsdeutsch in der Regel gar nicht oder allenfalls an marginaler Stelle.

„Die Wirtschaftssprache als homogenes Ganzes gibt es nicht“ (Hundt 1995, 8), seine umfassende Charakterisierung „erweist sich u. a. deshalb als sehr problematisch, da ,Wirtschaft' ein sehr heterogener Begriff ist" (Horst 1998, 49).

Eine der Wirtschaftssprachen, die von gegenwärtigen Fachsprachenforschern unter die Lupe genommen wird, ist die Sprache der Börse:

Für den Außenstehenden verdecken in den Kommentaren kollektive Substantive die Urheber des Geschehens und deren Interessen. Zu diesem ideologiekritisch als Verschleierung der ökonomischen und gesellschaftlichen Herrschaftsverhältnisse beschreibbaren Sprachgebrauch treten ergänzend metaphorische Bezeichnungen, die diesen Eindruck verstärken (Fluck 1996, 61f.).

Im Bereich der Börsenfachsprache, so wie es auch in anderen Fachsprachen der Fall ist, kommt es oft zu Verständigungshindernissen. Auf dieses Problem weist Fluck hin, indem er schreibt:

Die Sprache ist so hochverdichtet, dass sie fast nur für Eingeweihte, die Börsianer, zu verstehen, ist. Die Anhäufung von Fachtermini wie Aktie, Brief, Industrieanleihe, Kassakurse oder Lombardsatz setzt weitreichende Sachkenntnisse voraus. (Fluck 1996, 61f.).

Die genannte Verdichtung dieser Fachsprache hängt eng mit dem Metaphernreichtum der Fachsprache der Börse zusammen. Es soll jedoch betont werden, die metaphorische Ausdrucksweise ist nicht nur für die journalistische Wirtschaftssprache, sondern auch für die Wissenschafts- und Institutionssprache kennzeichnend (vgl. Hundt 1998, 110). Die Frage nach der Funktion von Metaphern in der Sprache des Wirtschaftsjournalismus verdient es - allein wegen der Frequenz, mit der sie gestellt wird - als ein selbständiges Forschungsvorhaben angesehen zu werden.

Der Terminus Metapher wird hauptsächlich als Stilmittel sowohl in der Lyrik als auch in der Rhetorik assoziiert. Er findet aber in der Sprachwissenschaft auch eine große Beachtung. Viele Autoren werten die Metapher als ein rein sprachliches Phänomen, die Linguisten Lakoff und Johnson sind jedoch der Meinung, 
dass unsere Handlungen, Gedanken und Einstellungen metaphorisch geprägt und strukturiert sind (vgl. Peter 2015, 3). Der Fokus dieses Textes liegt auf dem Begriff der konzeptuellen Metaphern, den Lakoff und Johnson geforscht und beschrieben haben. Dieser wird zunächst im theoretischen Teil ausführlich definiert und die damit verbundene Metaphernklassifizierung aufgedeckt, um darzustellen, inwieweit Metaphern die finanzielle Welt bestimmen. Infolgedessen wird die Relevanz der Metaphern in der Börsensprache beschrieben. Die prägnantesten Metaphern werden zunächst aufgelistet und anschließend auf damit verbundene metaphorische Konzepte und Klassifizierungen analysiert. Ebenfalls wird untersucht, wie bestimmte Aspekte in den Fokus rücken oder verschleiert werden. Zu guter Letzt erfolgt das Fazit, in dem alle genannten Themenpunkte noch einmal zusammengefasst und die relevantesten hervorgehoben werden.

\section{Die Entwicklungslinien der Metapherntheorie}

\subsection{Die (nicht)kognitiven Metapherntheorien}

Der Begriff Metapher findet seinen Ursprung in der westlichen Tradition zum ersten Mal bei Platon, der ihn der Rhetorik zuordnet. Eine spezifische Abhandlung darüber hat der griechische Philosoph jedoch nicht hinterlassen. Erst Platons Schüler Aristoteles verfasste die erste uns überlieferte Metapherntheorie, welche später Substitutionstheorie genannt wurde. Er beschreibt die Metapher in erster Linie im Blickfeld der Poetik als Instrument der Wissensvermittlung und zusätzlich, im Blickfeld der Rhetorik, als wichtige Komponente in der Formulierung aussagekräftiger Argumente (vgl. Schiefer 2006, 10). Er vertritt aber auch die Ansicht, dass

Metapher die Übertragung eines fremden Nomens ist entweder von der Gattung auf die Art oder von der Art auf die Gattung oder von einer Art auf eine andere oder gemäß der Analogie (Führmann 1979, 67).

Dieser Theorie zufolge ist die Metapher eine Substitution eines eigentlich auf einen gemeinten Referenten zutreffendes Wortes durch ein fremdes, also uneigentliches. Zwischen beiden Wörtern existiert eine Ähnlichkeit oder Analogie. Kurz $(1997,9)$ spricht an dieser Stelle von einem topologischen Modell der Sprache.

Diesem Modell zufolge kann für jedes Wort ein Ort angegeben werden, der ihm und nur ihm gehört (...). Ein Wort hat nur einen Ort, daher nur eine Bedeutung. Es gehört an diesen Ort, genauer: es gehört diesem Ort (...) (vgl. Kurz 1997, 8f). Noch eindeutiger als Aristoteles hat die spätere Rhetorik daraus die Lehre vom verbum proprium, dem, eigentlichen' Wort, entwickelt. Die Bedeutung eines Wortes wird dabei identifiziert mit dem Gegenstand, den es bezeich- 
net. (...) Die Metapher ist also die Ortsveränderung eines Nomens. Es wird von einem Ort auf einen anderen übertragen, dem es nicht gehört, dem es nicht eigen ist. Insofern hat die Metapher eine uneigentliche Bedeutung. Das übertragene Nomen bleibt transparent für seinen ursprünglichen Ort und für das Nomen, das eigentlich an den von der Metapher eingenommenen Platz gehört. In diesem topologischen Modell ist die Metapher also ein Akt des Ersetzens, des Borgens, Entlehnens und Entfremdens, ein Akt der Enteignung. Daher verwundert es nicht, dass die Metapher geradezu moralisch kritisiert werden konnte. Die Kritik der Metapher, vorgetragen bis ins 20. Jahrhundert hinein, setzt das aristotelisch-rhetorische Erklärungsmodell voraus. Nach dieser Kritik ist die Metapher keine authentische Bezeichnung, sie ist deplaziert, unernst und, weil nicht mehr eindeutig, ungenau und zweideutig. (vgl. Kurz 1997)

Daraus resultieren eine Falschaussage und der eben erwähnte Verfremdungseffekt, denn Achill ist natürlich kein Löwe, sondern der schnellste Läufer der Antike. Noch heute orientieren sich viele Metapherntheorien am Modell Aristoteles', wie unter anderem die Vergleichstheorie, die in der Metapher einen um die Partikel „wie“verkürzten Vergleich sieht (vgl. Fischer 2005, 11).

Im 17. Jahrhundert wird durch den Italiener Giambattista Vico eine Wende in der erkenntnistheoretischen Fundierung der Metapher und dem darauffolgenden philosophischen Denken eingeleitet. Er fechtet die Meinung der Grammatiker an, die besagt, dass die nüchterne Sprache der Wissenschaft die „eigentliche" und die Sprache der Poesie die uneigentliche" sei. Henri Bergson warnt davor, sich nicht von der angeblich nicht bildlichen Sprache der Wissenschaft blenden zu lassen, denn oft verstecke sich hinter abstrakter Sprache eine unbewusste, verräumlichende Metapher. Neben Vico tragen zahlreiche Philosophen und Dichter wie Johann Wolfgang von Goethe, Jean Paul, Gustav Gerber, Alfred Biese, Friedrich Nietzsche und Gertrude Buck dazu bei, eine Psychologie und Philosophie der Metapher zu entwerfen, in der diese nicht nur als schön, sondern auch für die Entwicklung und Struktur von Gedanken als elementar betrachtet wird (vgl. Schiefer 2006, 10-11). Eine Präzisierung dessen, was eine Metapher ist, scheint also schwierig (vgl. Schmitt 1995, 2011a). Lieb (1964) hat 125 verschiedene Definitionen seit der Antike rekonstruiert. Weinrich schlägt vor, „,alle Arten des sprachlichen Bildes von der Alltagsmetapher bis zum poetischen Symbol“ $(1967,7)$ zuzulassen. Ebenso skeptisch ist Black gegenüber einer operationalisierbaren Definition:

Metapher ist bestenfalls ein unscharfer Begriff, und wir müssen uns davor hüten, ihn strengeren Verwendungsregeln zu unterwerfen als in der Praxis tatsächlich zu finden sind (Black 1983, 59).

Newmark $(1985,298)$ beschreibt die Metaphern durch das Zusammenwirken der Kategorien object (das beschriebene Objekt), image (Bild/Gegenstand 
mit dem das Objekt umschrieben wird), sense (Sinn, Ähnlichkeiten zwischen Objekt und Bild), wogegen Snell-Hornby mit Bezug zur Blacks Interaktionstheorie die Metapher so versteht, dass sie „bestimmte Merkmale eines Gegenstandes selektiv auf einen anderen projiziert, wobei beide interagieren" (vgl. Schäffner 2006, 281). Die Metapher ist das Zusammenbringen zweier Vorstellungen in einen gemeinsamen aktiven Zusammenhang. Die Bindung vom sprachlichen Begriff wird gelöst, indem die Frage nach dem, Wie der Sprache ‘ auf das ,Wie des Denkens` erweitert wird (vgl. Richards 1936, 33ff.). Die Metapher ist demnach eine Doppeleinheit aus dem Hauptgegenstand, dem ,Tenor ' (tenor), und dem Beschreibenden, dem ,Vehikel' (vehicle).

Eine neue Sichtweise erlaubte sich Harald Weinrich. Er gilt als Begründer der sogenannten Bildfeldmetapherntheorie. In dem 1967 erschienenen Text Die Semantik der Metapher versucht er, die Metapher auf ihre Semantik hin zu definieren. Dabei sieht er die rhetorische Erklärung, die Metapher sei wie ein verkürztes Gleichnis, als „bequeme Definition“. Zunächst versucht er, die Metapher zu lokalisieren. Er ist der Ansicht, dass die Metapher bei der Zerlegung eines Satzes in seine einzelnen Wörter nicht an einem einzigen Wort festgemacht werden kann. Der Kontext, in dem sich die Wörter befinden, erschafft die Metapher. An dieser Stelle spricht Weinrich von dem Begriff des Bedeutungsumfangs eines Wortes. Um nun zu verstehen, was mit dem benutzten Wort genau gemeint ist, muss man es in den Kontext der anderen Wörter und des gesamten Textes setzen. Sie determinieren sich gegenseitig, schränken also die Zahl an möglichen Bedeutungen ein. Im Unterschied zu Metaphern können Wörter jedoch auch alleinexistieren, Metaphern benötigen einen Kontext (vgl. Weinrich 1976, 319). Den machen nicht nur verschiedene Wörter, sondern auch Situationen und einzelne Wörter durch die Wortsemantik aus. Der Kontext kann also auch innerhalb von Wörtern entstehen und sie zu Metaphern machen:

Eine Metapher ist ein Wort in einem Kontext, durch den es etwas anderes meint, als es bedeutet $[\ldots]$ wobei der ganze Kontext zu berücksichtigen (ist), und zwar ein Kontext, der nicht zu knapp bemessen ist. Ein Lendenschurz an Kontext genügt nicht (Weinrich 1963, 311f.).

\subsection{Die kognitive Metapherntheorie}

Die oben genannten Theorien zeichnen die Grundentwicklungslinien der Metapherntheorie. Jedoch erst in den 80er Jahren des 20. Jahrhunderts entwickelte sich die Metapher zu einer weiteren Stufe. In einer kognitiv-linguistischen Definition ist sie kein rein sprachliches Phänomen, sondern ist, ähnlich wie bei Black und Weinrich, auf der Ebene des Denkens angesiedelt, d. h. ein Erfahrungsbereich wird in Form eines anderen Erfahrungsbereiches verstanden 
(Lakoff/Johnson 1980; Lakoff/Johnson 1999). Das Verdienst von Lakoff und Johnson ist es, die Systematisierungsleistung der konzeptuellen Metaphern herausgearbeitet zu haben. Bei ihnen findet keine Diskussion über zwei Glieder der Metapher mehr statt. Ihre Grundidee kommt zwar von der Interaktionstheorie, doch ihre Perspektive ist eine neue. Seit dieser Zeit ist aber zu vermerken, dass die metaphorisch orientierten Arbeiten aus einer anderen Perspektive betrachtet werden und sich kaum noch mit rein rhetorischen oder literarischen Aspekten beschäftigen. Metaphern werden nicht nur als sprachliches Stilmittel und rhetorischer Schmuck angesehen, sondern als kognitives Phänomen. In der kognitiven Semantik, die unter dem Einfluss der Kognitionswissenschaft entstanden ist, hält man die Metapher für ein sprachliches Gebilde, das im Alltagsleben gebraucht wird und Teil unserer gedanklichen und sprachlichen Fähigkeit ist (vgl. Corbacho Sanchez 2009, 2f.). Als Ausgangspunkt der von Lakoff und Johnson entwickelten kognitiven Metapherntheorie gilt tatsächlich, dass Metaphern nicht nur die Sprache strukturieren, sondern vor allem ein wichtiger Bestandteil kognitiver Prozesse im menschlichen Denken und Handeln sind (vgl. Lakoff/Johnson 2007, 11). Die Metapher entsteht durch unsere Interaktion mit der Welt und so bildet sich unser konzeptuelles System. Den Mechanismus, mit dem ein abstraktes Konzept in Begriffen des Konkreten konzipiert wird, nennt man konzeptuelle Metapher. Hier befindet sich der Kern von Lakoffs und Johnsons Theorie, und um es mit ihren Worten zu sagen:

Das Wesen der Metapher besteht darin, dass wir durch sie eine Sache oder einen Vorgang in Begriffen einer anderen Sache bzw. eines anderen Vorgangs verstehen und erfahren können (Lakoff/Johnson 2007, 13).

Sogar die einfachsten Alltagshandlungen werden durch unsere metaphorischen Konzeptsysteme bestimmt. Dabei ist die Verbalisierung der Ausdruck (metaphorische Äußerungen) dieser Konzeptsysteme.

Wir haben $[\ldots]$ festgestellt, dass die Metapher unser Alltagsleben durchdringt, und zwar nicht nur unsere Sprache, sondern auch unser Denken und Handeln. Unser alltägliches Konzeptsystem, nach dem wir sowohl denken als auch handeln, ist im Kern und grundsätzlich metaphorisch (Lakoff/Johnson 1998, 11).

Eine Metapher wird in diesem Sinne als ein kognitives Phänomen angesehen, welches menschliches Denken und Handeln organisiert und strukturiert: „The metaphor is not just a matter of language, but of thought and reason" (Lakoff 1993, 208) bzw. „a mode of thought“ (ebd., 210) - sie stellt also primär ein Denkphänomen dar, welches sich erst sekundär in der Sprache niederschlägt. Dabei zeichnet sich eine konzeptuelle Metapher dadurch aus, „dass wir durch sie eine Sache oder einen Vorgang in Begriffen einer anderen Sache bzw. eines anderen Vorgangs 
verstehen und erfahren können“ (Lakoff/Johnson 2007, 13). Eine Metapher beschreibt dabei eine Verknüpfung von zwei unterschiedlichen Konzepten bzw. konzeptuellen Domänen. Die meisten alltäglich verwendeten Metaphern sind in hohem Maße konventionalisiert, sodass sie häufig gar nicht als solche erkannt werden (vgl. Lakoff/Johnson 2007, 11f.). Darüber hinaus ist die Struktur des menschlichen Konzeptsystems zum größten Teil nicht bewusst erfahrbar. Die meisten kognitiven Prozesse vollziehen sich weitestgehend - etwa zu 80 Prozent - unbewusst und unreflektiert (vgl. Lakoff/Wehling 2008, 22f.). Mit anderen Worten, nur wenn wir mit Hilfe der sprachlichen Oberflächenstruktur die konzeptuelle Tiefenstruktur zum Vorschein bringen, „können wir anhand von metaphorischen sprachlichen Ausdrücken das Wesen metaphorischer Konzepte untersuchen und Einsicht gewinnen in die metaphorische Natur unserer Aktivitäten“ (Lakoff/Johnson 2007, 15).

Die Prämissen dieser Theorie lassen sich also in folgenden Punkten zusammenfassen:

1. Das konzeptuelle System des Menschen ist weitgehend metaphorisch.

2. Die metaphorische Sprache ist zweitrangig und ist dem (konzeptuell-) Kognitiven untergeordnet.

3. Das kognitive (metaphorische) System bestimmt das Denken und die Wahrnehmung.

4. Das kognitive (metaphorische) System bestimmt das Handeln.

Gemäß der Ubiquitätsthese (vgl. Jäkel 1997, 40) ist das Phänomen der konzeptuellen Metapher nicht nur ein bedeutungsvoller Bestandteil der Alltagskommunikation, sondern auch der wissenschaftsbezogenen Fachsprache. Der kommunikative Kontext bestimmt auf jeden Fall, ob eine Redewendung metaphorisch gebraucht wird oder nicht. Aus dieser Perspektive gesehen ist der Bereich der Börsensprache auf keinen Fall frei von Metaphern (vgl. Corbacho Sanchez 2009, 3f). Die Wirtschaftsexperten drücken sich in ihren alltäglichen Gesprächen metaphorisch aus (vgl. Jäkel 1997, 93f).

\section{Metapher in der Sprache der Börse}

\subsection{Börsenfachsprache}

Die Börsensprache kann als Subsystem des Fachbereichs Finanzwesen eingestuft werden, das wiederum eine Spezifizierung des Sprachkomplexes Wirtschaft darstellt. Die Zugehörigkeit zum Finanzwesen ist durch die traditionellen Instrumente des Börsenhandels klar gegeben. Hinter der Geschäftsabwicklung an der Börse versteckt sich jedoch der ganze wirtschaftliche Hintergrund des Wertpapiergeschäfts - die betriebsinterne Situation und allgemeine Marktdaten. Einschließen muss man auch das gesellschaftliche Klima und die psychologischen 
Aspekte des menschlichen Handelns unter die Lupe nehmen. Deshalb weist die Lexik dieser Varietät stark fachübergreifende Tendenzen auf. Ein wichtiges Merkmal der Börsensprache ist ihre relative Isolierung. Die Geschäfte werden in dafür bestimmten Räumen abgewickelt, in die der Laie keinen Zutritt hat. Logischerweise muss sich die Öffentlichkeit bei der Vermittlung der Börseninformationen mehr auf den Informationsträger verlassen, in diesem Fall auf die Medien. Das interessierte Publikum bekommt so ein schon verarbeitetes Produkt der Sprache, dem mehr oder weniger die Regeln des journalistischen Stils aufgezwungen werden. Das ist ein wichtiges Merkmal, welches man besonders bei der Analyse der Zeitungsartikel berücksichtigen muss (vgl. Březina 2014, 28). Die Börsenfachsprache gehört zu den Fachsprachen, für die „ein hochfrequenter Gebrauch von terminologisierten Einheiten, die auf dem Wege entweder der metonymischen [...] oder metaphorischen Bezeichnungsübertragung $[\ldots]$ entstanden sind“ (Nycz 2009, 161) charakteristisch sind. Der Begriff Börsensprache bezieht sich aber vor allem auf die sogenannten Börsenberichte, die das Börsenklima zum Vorschein bringen. Die von Arnold durchgeführte Analyse weist darauf hin, dass die Fachkommunikation im Börsenwesen eine „Einschränkung $[\ldots]$ auf eine bestimmte soziale Gruppe (Wirtschaftsfachleute), ein Kommunikationsmedium (Zeitung) und einen Gegenstandsbereich (Wirtschaft)“ darstellt, was die „Ausbildung einer stark normierten Fachsprache zur Folge“ (Arnold 1973, 108) hat. Die Börsenberichte lassen sich in Bezug auf die Empfänger in drei Gruppen aufteilen: Fachleute bzw. professionelle Anleger oder Dienstleistungen, die mit der Börse vertraute, anspruchsvolle Leser und die Personen mit nur geringem Vorwissen über die Börse. Theoretisch gesehen soll ein unterschiedlicher Anteil von Termini in Berichten aufgrund der unterschiedlichen Empfängergruppen verzeichnet werden, leider hat sich diese Hypothese als unrecht erwiesen. Als Beleg zu nennen ist die Untersuchung von Nycz. Nach Analyse und Vergleich schlussfolgert er, dass die an drei Gruppen gerichteten Berichte „durch eine ähnlich hohe Frequenz von Termini gekennzeichnet" sind, „die sich in jedem Fall auf ca. 30\% der autosemantischen Wörter beläuft“ (Nycz 2009, 90). Man geht davon aus, dass die Sprachen in Börsenberichten angesichts ihrer Verdichtung von Fachtermini sehr fachspezifisch und nur für Eingeweihte oder Börsianer zugänglich sind (vgl. Fluck 1996, 61).

Die Börsenberichte sind durch eine starke Anhäufung von Fachtermini im engeren Sinne geprägt, deren Verständnis erhebliche Sachkenntnis erfordert. Fachsprache im weiteren Sinne ist fast der gesamte Sprachgebrauch der Börsennachrichten, denn nahezu sämtliche Sprachelemente sind Formeln mit einem bestimmten Sinn fixiert (vgl. Arnold 1973, 104). 


\subsection{Metaphorik der Börsensprache}

Die Fachlichkeit der Börsenberichte besteht zudem in der Anhäufung von Metaphern. Im Vergleich zu anderen Fachbereichen werden jeder Zeit die aktuellsten Informationen über die Börse durch Übertragungskanäle, vor allem Internet, dem Publikum weitergegeben. Um die fachlichen Inhalte zugänglicher zu machen, wurden viele durch konzeptuelle Übertragung entstehende Metaphern in die Berichte eingesetzt, was dann im Laufe der Zeit zu einem der wichtigsten Merkmalen der Börsensprache geworden ist. Nach Fluck (1996) vermitteln „die meisten dieser metaphorischen Termini den Eindruck von Bewegung und Dynamik, der durch den Gebrauch von Tätigkeits- und Vorgangsverben noch erhöht wird" (Fluck 1996, 62). Im Kontext der Börsenberichte sind die Metaphern insoweit wichtig, weil sie „in anderen Fachsprachen, wie z. B. in der Sprache der Wirtschaftstheorie, kaum vorstellbar wären" (Nycz 2009, 161). Winko (2006, 18) hebt die kommunikative Rolle der Metapher hervor, die ihr zufolge verursacht, dass Metaphern in den Börsenberichten so oft eingesetzt werden, um über Emotionen zu sprechen:

a) Metaphern beziehen sich auf Sachverhalte, Wahrnehmungen oder Zustände, die wörtlich nicht oder nur umständlich zu benennen sind,

b) Metaphern bezeichnen ihren Gegenstand in kompakter, kondensierter Form, enthalten also viele Information auf wenig Raum,

c) Metaphern drücken Sachverhalte, Wahrnehmungen oder Zustände anschaulicher und „lebhafter" aus als nicht-metaphorische Ausdrücke, und zwar mittels detaillierterer und reicherer Information. Sie werden alltagssprachlich benutzt und bilden so ein perfektes Mittel, um spezifische Emotionen zu bezeichnen oder auszudrücken.

Die Untersuchungen der Metaphorik in den deutschen Wirtschaftskommentaren, die Schoenke $(1996,22)$ vorgelegt hat, deuten auf einen starken Einsatz von Metaphern hin. Schoenke betont den konzeptuellen Charakter dieser Metaphern. Als wichtiges Merkmal hebt sie hervor, „dass die besonders in den Überschriften benutzten Metaphern oft eine verschlüsselte, verrätselte Stellungnahme enthalten, deren Bezug zum bewerteten Sachverhalt nur sehr schwierig oder gar nicht zu erkennen ist" (Schoenke 1996, 105).

In Bezug auf Verbreitung und Frequenz von Metaphern hebt Pytelka in den Börsenberichten folgende Themenbereiche hervor:

1) das Börsengeschehen: die Preisbewegung und die Abwicklung der Börsengeschäfte,

2) die Charakterisierung der Geschäfte, der Situation an der Börse, der Wertpapiere,

3) die Börsenmitglieder, die Stimmung an der Börse, die Arten von Börsengeschäften (vgl. Pytelka 1971, 101). 
In dem vorliegenden Beitrag wurden Metaphern der deutschen Börsenberichte unter die Lupe genommen. Welche Herkunftsbereiche favorisiert werden, ergibt sich aus mehreren Texten der online veröffentlichten Börsenberichte (u. a. boerse.de, finanzen.de, finanzen.net). In der Analyse sollen die Metaphern auf ihre verschiedenen Kontexte, bzw. Bildspenderbereiche genauer untersucht werden. In Bezug auf den Zielbereich Börse liefert der bildspendende Bereich Krieg die meisten metaphorischen Ausdrücke. Auf den weiteren Plätzen erscheinen metaphorische Ausdrücke aus den Themenbereichen Sport, Mensch oder Gebirge. Da Krieg als Motiv schon mehrmals unter die Lupe genommen wurde (vgl. Stawikowska-Marcinkowska 2016, 163), wurden die drei letzten zum Gegenstand des empirischen Teils des Beitrags.

\subsubsection{Bildspenderbereich Sport}

Die Börse wird sehr oft als Spielfeld der Finanzen bezeichnet. Auf einem Spielfeld besteht die Erwartung, andere zu übertrumpfen, Erster zu sein, den Wettkampf zu gewinnen bzw. im Vordergrund zu stehen. Aus diesem Grund stellt auch die Sportmetapher eine sehr ergiebige Quelle zur Erschließung der Börsengeschehnisse dar und findet eine breite Verwendung in der Börsensprache. Im Sport steht das friedliche und in Regelwerken festgehaltene Kräftemessen im Vordergrund. Die Sportler wollen in einer Kraftprobe ihre Leistung, mit dem Ziel, zu gewinnen, demonstrieren. Projiziert man dies auf die Börse, so findet ein Kräftemessen zwischen Börsen, Aktienindizes und börsennotierten Unternehmen statt. Beispiele ${ }^{1}$ :

Der Dax hat zum Wochenauftakt einen weiteren Schritt in Richtung Rekordhoch getan. Zum Handelsschluss am Montag stand der deutsche Leitindex 0,22 Prozent im Plus bei 13 463,69 Punkten. (boerse.de, 15.10.2018)

Einen Rekord mit 12 317,00 Punkten erreichte im frühen Handel der Kleinwerteindex SDax, der zuletzt bei 12 299,82 Punkten und einem Plus von 0,33 Prozent notierte. (boerse.de, 10.09.2018)

Dax könnte 13000 Punkte überspringen. (boerse.de, 10.05.2018)

Unterstützt von saudischen Investoren ebnet Vertex das Spielfeld, indem sie potenzielle Stakeholder bei der Beteiligung an Investitionen unterstützen. (finanzen.net, 30.09.2018)

Die große Abwärtskurslücke im Tageschart vom März (46,43 USD zu 49,70 USD) definiere dabei ein erstes Etappenziel, ehe die 200-Tage-Linie (akt. bei 51,29 USD) in den Fokus rücke. Um das diskutierte Sprungbrett nicht zu gefährden, gelte es zukünftig die Bastion bei rund 40 USD zu verteidigen, so die Analysten von HSBC Trinkaus \& Burkhardt in einer aktuellen Chartanalyse. (boerse.de, 15.06.2018)

${ }^{1}$ In allen nachstehenden Beispielen stammen die Hervorhebungen von der Autorin. 


\subsubsection{Bildspenderbereich Mensch}

Eine ebenfalls häufig verwendete Quelle metaphorischer Übertragungen auf das Konzept Börse ist der Bildspender Mensch, der teilweise in den Bereich der Personifizierung fällt. Hierbei werden für den Menschen typische Tätigkeiten, Charakteristika und Fähigkeiten auf die Börse und ihre Abläufe projiziert, wobei die Aktien, Unternehmer und das ganze Börsengeschehen menschliche Attribute entfalten. Beispiele:

Der Küchenbauer Alno will 2019 finanziell wieder auf eigenen Beinen stehen und dann in die Gewinnzone kommen. (boerse.de, 15.01.2018)

Der dafür notwendige schrittweise Ausstieg aus der Kohleverstromung würde RWE belasten - und das in einer Zeit, in der die Höhe des Mittelzuflusses seitens der Ökostromtochter Innogy auf wackeligen Füßen stehe. (boerse.de, 30.01.2018)

Die Luft ist nicht raus. Der Dax zum Beispiel hat noch gut 500 Punkte Vorsprung aufseinen GD200, den gleitenden Durchschnittskurs. So sehen Trendfolger die Situation. Dabei orientiert sich unsere hauseigene Investitionsquote an den Abständen zur 200-Tage-Linie. Also ist ordentlich Luft nach unten - und oben. Das genaue Gegenteil davon, dass die Luft draussen ist. Es wird spannend. (boerse.de, 26.06.2014)

Die deutschen Aktienindizes sind am Freitag freundlich gestartet. Insgesamt ist der Handel jedoch von schmalen Umsätzen geprägt. (finanzen.de, 13.04.2017)

\subsubsection{Bildspenderbereich Gebirge}

Eine metaphorische Durchdringung der Börsenwelt über den Rückgriff auf die Gebirgsmetapher findet häufig statt. So weisen vor allem die metaphorischen Auf- und Abwärtsbewegungen darauf hin, dass sich die Börse als eine Gebirgslandschaft konzeptualisieren lässt, in der die am Börsengeschehen beteiligten Komponenten bestrebt sind, den Gipfel zu erklimmen. Beispiele:

Activision Blizzard-Aktie klettert leicht um 0,7\%. (boerse.de, 12.10.2018)

Die Kursrally der vergangenen Monate wie auch der DAX-Aufstieg sind aber nicht nur positiv zu sehen. So sollten sich Anleger positionieren. (finanzen.de, 17.09.2018)

SAP: Neue Höhen erklommen. Für Rückenwind sorgte das starke Marktumfeld der Techwerte. Die US-Technologieindizes Nasdaq 100 und Nasdaq Composite kletterten ebenfalls auf neue Rekordstände. (finanzen.net, 18.06.2018)

US-Banken könnten den Gipfel der Profite bald erreichen. (handelsblatt.de, 11.10.2018)

Der Euro hat am Donnerstag seine Talfahrt der vergangenen Handelstage vorerst gestoppt und zu einer Erholung angesetzt. Zu Wochenmitte war der Kurs zeitweise bis auf 1,1301 US-Dollar gefallen und damit auf den tiefsten Stand seit Juni 2017. (finanzen. net, 16.08.2018) 


\section{Schlussfolgerungen}

Die aktuelle Metaphernforschung geht davon aus, dass die menschliche Wahrnehmung der Welt vorwiegend metaphorischer Natur ist. Dieser Beitrag befasst sich in erster Linie mit dem Problem der Begriffsbestimmung des Metaphernbegriffs innerhalb eines kognitiv-linguistischen Paradigmas. Zur Differenzierung des Metaphernbegriffs wurden exemplarisch theoretische Ansätze vorgestellt, die zeitlich vor der Conceptual Metaphor Theory liegen und bereits in unterschiedlichem Grad wichtige Kernpunkte eines kognitiven Metaphernbegriffs vorwegnehmen. Dieser kognitive Metaphernbegriff ist schließlich anhand der Theorie von Lakoff und Johnson dargestellt worden. Die empirische Untersuchung gab einen Einblick in die Vielfalt metaphorischer Konzeptualisierung der Börsendomäne, kann aber aufgrund der notwendigen Einschränkung der Korpusgrundlage keinen Anspruch auf Vollständigkeit erheben. Nach Auffassung von Lakoff und Johnson dienen die Metaphern der Veranschaulichung abstrakter Prozesse und komplexer Zusammenhänge, indem sie auf vertraute Konzepte zurückgreifen. Damit lassen sich abstrakte Wissensgebiete, wie das der Börse, verständlicher darstellen. Demnach kann man den Metaphern in den wirtschaftlichen Texten drei Funktionen zuordnen:

1. Terminologie bildende Funktion: mithilfe von Metaphern ermöglicht die Entstehung neuer Termini

2. Stilistische Funktion - es wird mit Hilfe von überwiegend konventionalisierten und klischeehaften Bezeichnungen mehr auf die Wirkung und Abwechslung geachtet.

3. Pragmatische Funktion - metaphorische Ausdrücke dienen dazu, harte und unangenehme Tatsachen zu mildern oder zu verschönern, um die Informationsempfänger zu beeinflussen.

Was aber bei der Untersuchung zur Ansicht kam, ist die Tatsache, dass viele Metaphern in der Sprachgemeinschaft so stark integriert sind, dass sie nicht mehr als Metaphern wahrgenommen werden. Sie werden als konventionelle oder auch ,tote“ Metaphern bezeichnet (vgl. Jäkel 2003, 50f.).

\section{Literatur}

Arnold, Volker (1973): Kritische Analyse des Sprachgebrauchs der Wirtschaftsjournalistik in Tageszeitungen. In: Projekt Deutschunterricht. Bd. 4. Stuttgart, S. 94-119.

Aristoteles (1979): Poetik. Griechisch/Deutsch. Übersetzt und herausgegeben von Manfred Fuhrmann, Stuttgart, Kap. 21, 1457b.

Black, Max (1983): Die Metapher (1954). In: Anselm Haverkamp, Hrsg. Theorie der Metapher. Darmstadt, S. 55-79. 
Bolten, Jürgen (1991): Fremdsprache Wirtschaftdeutsch: Bestandsaufnahme und Perspektiven. In: Bernd-Dietrich Müller, Hrsg. Interkulturelle Wirtschaftskommunikation. München: Iudicum, S. 71-91.

Březina, Jaroslav (2004): Phänomen Fachsprachen - Börsensprache unter der Lupe, in: Philologie im Netz Herausgegeben von Paul Gévaudan, Hiltrud Lautenbach, Alexander Nebrig, Peter Schneck und Dietrich Scholler, S. 17-38.

Corbacho Sánchez, Alfonso. Zur Wassermetaphorik in Phraseologismen der Wirtschaftssprache. Eine kognitive Studie anhand des Deutschen und Spanischen. Revista de Lingüística y Lenguas Aplicadas, [S. 1.], v. 4, p. 37-45, oct. 2010. ISSN 1886-6298. Available at: https://polipapers.upv.es/index.php/rdlyla/article/view/732. Date accessed: 05 july 2020. doi: https://doi.org/10.4995/rlyla.2009.732.

Fischer, Hans Rudi (2005): Eine Rose ist eine Rose. Zur Rolle und Funktion von Metaphern in Wissenschaft und Therapie. Weilerswist.

Fluck, Hans-Rüdiger (1996): Fachsprachen. Einführung und Bibliographie. Tübingen/ Basel: Francke.

Horst, Sabine (1998): Wortbildung in der deutschen Wirtschaftskommunikation. Linguistische Modelle und fremdsprachdidaktische Perspektiven. Waldsteinberg: Heidrun Popp Verlag.

Hundt, Markus (1995): Modellbildung in der Wirtschaftssprache. Zur Geschichte der Institutionen und Theoriefachsprachen der Wirtschaft. Tübingen: Max Niemeyer Verlag.

Hundt, Markus (1998): Typologien der Wirtschaftssprache: Spekulation oder Notwendigkeit? In: Fachsprache 20, Heft 3-4, 98-115.

Jäkel, Olaf (1997): Metaphern in abstrakten Diskurs-Domänen. Eine kognitive-linguistische Untersuchung der Bereiche Geistestätigkeit, Wirtschaft und Wissenschaft. Frankfurt am Main.

Jäkel, Olaf (2003): Wie Metaphern Wissen schaffen, Verlag Dr. Kovač in Hamburg.

Lang KURZ, Gerhard (1997): Metapher, Allegorie, Symbol. Göttingen, 4. Aufl.

Lakoff, George/Johnson, Mark (1980): Metaphors We Live By. Chicago \& London.

Lakoff, George/Johnson, Mark (1999): Philosophy in The Flesh. The Embodied Mind and Its Challenge to Western Thought. New York.

Lakoff, George/Johnson, Mark (2007): Leben in Metaphern, Carl-Auer-Verlag, Heidelberg.

Lakoff, George/Wehling, Elisabeth (2008): Auf leisen Sohlen ins Gehirn: Politische Sprache und ihre heimliche Macht (Tiptoeing into the brain: Political language and its secret powers). Heidelberg: Carl-Auer.

Lieb, Hans-Heinrich (1964): Der Umfang des historischen Metaphernbegriffs. Dissertation Köln.

Newmark, Peter (1985): The Translation of Metaphor. In: W. Paprott/R. Dirven (Hg.): The Ubiquity of Metaphor. Amsterdam, S. 295-326.

Nycz, Krzysztof (2009): Fachterminologie als Mittel des Fachwissenstransfers: Dargestellt am Beispiel der deutschen Börsenberichterstattung. Hamburg.

Peter, Jacqueline (2015): Eine Analyse Konzeptueller Metaphern in der Medizin nach Lakoff und Johnson anhand eines Interviewtranskripts. Heine-Heinrich-Universität-Düsseldorf, Hausarbeiten. 
Pytelka, Josef (1971): Die Metaphern in der Sprache der englischen Börsenberichte. In: Lebende Sprachen, 16/6. Berlin.

Schäffner, Christina (2006): Metaphern. In: Handbuch Translation (2). Snell-Hornby, Mary; Hönig, Hans G.; Kußmaul, Paul (Hrsg.), S. 280ff.

Schiefer, Mattthias (2006): Die metaphorische Sprache in der Medizin. Metaphorische Konzeptualisierungen in der Medizin und ihre ethischen Implikationen untersucht anhand von Arztbriefanalysen. Wien.

Schmitt, Rudolf (1995): Metaphern des Helfens. Weinheim: Psychologie Verlags-Union.

Schoenke, Eva (1996): Titel und Themenentfaltung in Wirtschaftskommentaren. In: Schoenke, Eva (Hrsg.), Wirtschaftskommentare - textlinguistische Analysen - kontrastive Untersuchungen, S. 11-52.

Stawikowska-Marcinkowska, Agnieszka (2016): Eine Vergleichsstudie zur Metapher in den deutschen und polnischen Börsenberichten Kommunikationsformen in der Fach- und Gemeinsprache. Felder der Sprache - Felder der Forschung. Lodzer Germanistikbeiträge 7. Primum Verbum, Łódź.

Weinrich, Harald (1963): Semantik der kühnen Metapher. In: Sprache in Texten, S. 295-316.

Weinrich, Harald (1967): Allgemeine Semantik der Metapher. In: Sprache in Texten, S. 317-327.

Weinrich, Harald (1976): Sprache in Texten, Stuttgart.

Winko, Simone (2006): Wovon man kaum sprechen kann. Emotionen und Metaphern in literarischen Texten. In: Der Deutschunterricht 6, S. 18-25. 